\title{
Extra Dose Measurement of Differential Slice Thickness of MVCT Image with Helical Tomotherapy
}

\author{
Byungkoo Lee*, Suman Kang** \\ Department of Radiation Oncology, Korea University Anam Hospital, \\ Department of Radiological Science, Dong-eui University ${ }^{* *}$
}

\section{토모테라피 치료 시 MVCT Image의 Slice Thickness 차이에 따른 선량 비교}

\author{
이병구*, 강수만** \\ 고려대학교 안암병원 방사선 종양학과*, 동의대학교 방사선학과**
}

\begin{abstract}
Helical Tomotherapy is an innovative means of delivering intensity modulated radiation therapy (IMRT) using a device that merges features of a linear accelerator and helical computed tomography (CT) scanner. Hereat, during helical tomotherapy process, megavoltage computed tomography (MVCT) image are usually used for guiding the precise set-up of patient before/after treatment delivery. But which would certainly increase the total dose for patients, this study was to investigate the imaging dose of MVCT using the cylindrical "Cheese" phantom on a tomotherapy machine. A set of cylindrical "Cheese" phantom was adopted for scanning with respectively pitch value $(1,2,3 \mathrm{~mm})$ with same number slice (10 slice), same length (approximately $9 \mathrm{~cm}$ ) and phantom set-ups on the couch of tomotherapy system. The average MVCT imaging dose were measured using A1SL ion chamber inserted in the phantom with preset geometry.

The MVCT scanning average dose for the cylindrical "Cheese" phantom was 2.24 cGy, 1.02 cGy, 0.81 cGy during respectively pitch value (pitch $1,2,3 \mathrm{~mm}$ ) with same number slice (10 slice), and same length's average dose was $2.47 \mathrm{cGy}, 1.28 \mathrm{cGy}, 0.88 \mathrm{cGy}$ respectively (pitch 1, 2, $3 \mathrm{~mm}$ ). Two major parameters, the assigned pitch numbers and scanning length, where the most important impacts to the dose variation. The MVCT dose was inversely proportional to the CT pitch value. The results may provide a reliable guidance for proper planning design of the scanning region, which is valuable to help minimize the extra dose to patient.

Questionnaires were distributed to Radiology departments at hospitals with 300 sickbeds throughout the Pohang region of North Gyeongsang Province concerning awareness and performance levels of infection control. The investigation included measurements of the pollution levels of imaging equipment and assistive apparatuses in order to prepare a plan for the activation of prevention and management of hospital infections. The survey was designed to question respondents in regards to personal data, infection management prevention education, and infection management guidelines.
\end{abstract}

Key Words : Tomotherapy, MVCT, extra dose 


\section{요약}

의료용 선형가속기 (linear accelerator)와 나선형 컴퓨터 단층 촬영 장치 (helical computed tomography scanner) 의 결합 장치인 토모테라피는 세기 변조 방사선 치료 (intensity modulated radiation therapy(IMRT))의 큰 혁신을 이 끌었다. 토모테라피 치료 과정에서, Megavoltage computed tomography (MVCT) 영상획득은 치료 환자의 정확한 자 세 정렬을 위해 치료 전 또는 후에 이용된다. 그러나 이는 환자의 총 선량을 증가시키는 결과를 만들며, 본 연구는 이 처럼 MVCT 영상 획득 시 증가되는 선량을 Cylindrical "Cheese" Phantom을 이용하여 측정, 비교하였다. 각각의 pitch 별로 $(1,2,3 \mathrm{~mm}$ ) 동일한 개수의 slice (10 slice), 그리고 동일한 length (약 $9 \mathrm{~cm}$ )를 scanning하여, 이때의 선량 (MVCT Scanning Dose)을 A1SL ion chamber를 이용하여 측정하였다. 측정 결과 동일한 Slice 개수 (각각의 pitch 당 10 개)일 때, MVCT scanning dose의 평균값은 각각 (pitch 1,2,3mm) $2.24 \mathrm{cGy}, 1.02 \mathrm{cGy}, 0.81 \mathrm{cGy}$ 가 측정되었 다. 동일한 length에서 MVCT scanning dose의 평균값은 각각 (pitch 1,2,3mm) $2.47 \mathrm{cGy}, 1.28 \mathrm{cGy}, 0.88 \mathrm{cGy}$ 가 측정되었다. 이는 할당된 pitch와 scanning length가 MVCT scanning dose에 큰 영향을 미치는 가장 중요한 매개 변수 임을 말하며, pitch는 MVCT scanning dose와 역비례 관계를 나타냈다. 때문에 적절한 pitch와 scanning length의 선택 으로 치료 선량 외의 추가 선량을 최소로 줄여야 하겠다.

중심단어: 토모테라피, MVCT, 추가 선량

\section{I. 서론}

현재 많이 이용되고 있는 영상유도 기법 (Radiographic image guidance technique)은 환자의 자세 정렬, 표적의 위치 확인, 외부 조사 빔의 정렬 등에 이용되 고 있다. 이에 따라 많은 장치들이 개발, 발전되어 왔 으며, $\mathrm{kV}$ Cone-Beam CT, MV Cone-Beam CT, 그리고 MVCT를 이용한 Tomotherapy 등등이 그것이다. 그러 나 Aird 등은 외부 조사의 선량 전달과정에서 수반되 는 추가적인 선량을, 선형가속기의 누설선량과 산란 선, 그리고 체내에서 일차선과 산란선에 의해 표적 체 적 이외의 부위에 조사되는 선량, 뿐만 아니라 치료 선량과는 직접적인 관계가 없는 확인 영상을 위한 선 량 등으로 표현 하였고, 이러한 부가 선량은 전체 치 료 기간 중 영상 획득 과정이 많을수록 더욱 증가 한 다고 하였다 ${ }^{[1]}$.

또한 kilovoltage $(\mathrm{kV})$ 에서 megavoltage(MV)까지의 에 너지 범위에서 연부조직과의 주 상호작용은 콤프턴 산란이므로 $\mathrm{kVCT}$ 와 동일한 해상도와 signal to noise ratio(SNR)를 얻기 위해서 MVCT는 더 많은 선량이 필 요하다. 때문에 정확한 정보를 얻기 위한 영상의 질과 추가적인 피폭을 최소화 할 수 있는 선량의 선택은 심 각히 고려할 사항이라 할 수 있다.
Tomotherapy는 일반적인 선형가속기에 진단용 fan-beam CT scanner가 결합되어진 형태로, 정상조직과 중요 장기는 보호함과 동시에 종양조직에는 최대의 치료효과를 줄 수 있는 장점이 있다 ${ }^{[2]}$. 실질적인 환자 의 방사선치료 행위 전에 매번 MVCT image를 획득하 여, 이미 획득한 치료계획용의 $\mathrm{kVCT}$ image와 자세 정 렬 상태 등을 내, 외부적으로 비교, 보정함으로써 기존 의 방사선 치료보다 더욱 정교하고 세밀한 치료가 가 능하다. 이러한 장점에도 불구하고 매번 치료시마다 촬영하는 MVCT image는 진단용의 $\mathrm{kVCT}$ image가 아닌 치료 시 사용하는 고에너지 $(3.5 \mathrm{MeV}$, average energy of $1.36 \mathrm{MeV})^{[3],[4],[5]}$ 의 방사선을 사용하기 때문에 환자에 게 계획된 선량보다 더 많은 방사선이 들어가게 된다. 본 논문의 목적은 $\mathrm{MVCT}$ 촬영 시 사용하는 고정된 3 가지 protocol의 각 Slice Thickness (pitch $1 \mathrm{~mm}$, pitch 2 $\mathrm{mm}$, pitch $3 \mathrm{~mm}$ )에서의 Point dose 차이를 정량적으로 비교해봄으로써, 임상치료에서의 유용성 (Good Image Quality and Lower Absorbed dose)을 알아보고자 한다. 


\section{II. 대상 및 방법}

사용하는 장비로는 Tomotherapy사의 Hi-ART II와, 각 Slice 두께 (pitch $1 \mathrm{~mm}$, pitch $2 \mathrm{~mm}$, pitch $3 \mathrm{~mm}$ )에 따른 Point dose를 측정하고자 직경 $6.4 \mathrm{~mm}(0.250 ")$, Collecting volume $0.057 \mathrm{cc}$ 인 Exradian A1SL ion Chamber 를 사용하였다. 그리고 2 개의 반원 형태를 rubber ties 로 고정해, 직경 $30 \mathrm{~cm}$, 길이 $18 \mathrm{~cm}$ 의 원통 형태인 Cylindrical "Cheese" Phantom과 8 channel의 TomoElectrometer, 온도와 기압의 보정은 Lufft C-100, C-300 model(germany)을 사용하였다. [그림 1.]은 측정 장치의 배열을 보여 준다.

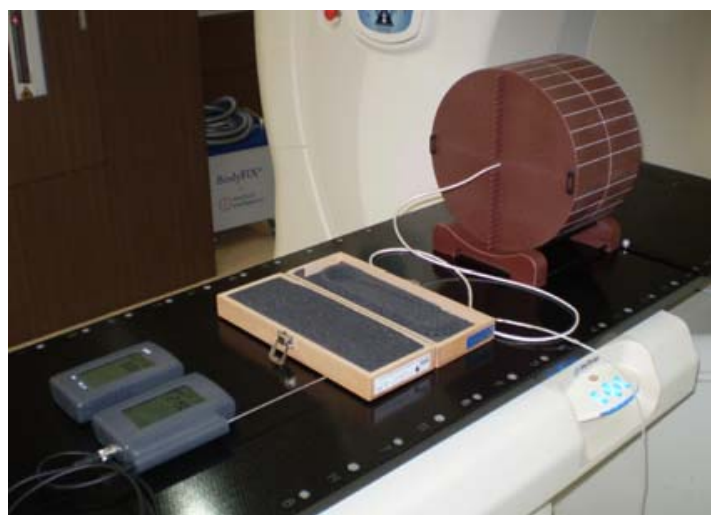

Fig.1. Measurement geometry

실제 치료 시 환자가 받는 선량을 직접 측정 할 수 없으므로, MSAD (Multiple scan average dose) 방법을 이 용하여 ion chamber가 직접 받는 선량과 주변 Slice의 scatter를 통합한 Point dose를 측정하였다 ${ }^{[6]}$. MSAD는 Phantom의 전체적인 Volume을 Scan 할 때, 중심부위의 평균적인 Point dose 값을 나타낸다 ${ }^{[7]}$.

실험은 두 가지 방법으로 나누어 실시하였다. 실제 환자 치료 전에 수행하는 DQA (Delivery Quality Assurance) Procedure를 이용하여, 첫째, 동일한 Slice 개 수(10 Slice)를 정하고 이에 각 Slice Thickness (pitch 1, 2, $3 \mathrm{~mm}$ )별로 측정, 선량차이를 비교하여, 총 3 번의 실험 을 통해 평균값을 구하였다. 즉, pitch $1 \mathrm{~mm}$, scan slice 두께 $2 \mathrm{~mm}$ 로 10 개의 slice를 scan 하였을 때, 총 scan length는 $2.0 \mathrm{~cm}$ 이며, pitch $2 \mathrm{~mm}$, scan slice 두께 $4 \mathrm{~mm}$ 로 10 개의 slice를 scan 하였을 때, 총 scan length는 4.0 $\mathrm{cm}$ 이고, pitch $3 \mathrm{~mm}$, scan slice 두께 $6 \mathrm{~mm}$ 로 10 개의 slice를 scan 하였을 때, 총 scan length는 $6.0 \mathrm{~cm}$ 이다. [그림 2.]는 pitch 별 scan slice의 두께를 나타내며, [그림 3.]은 10 개의 slice를 각 pitch 별로 scan 할 때의 모습이 다.

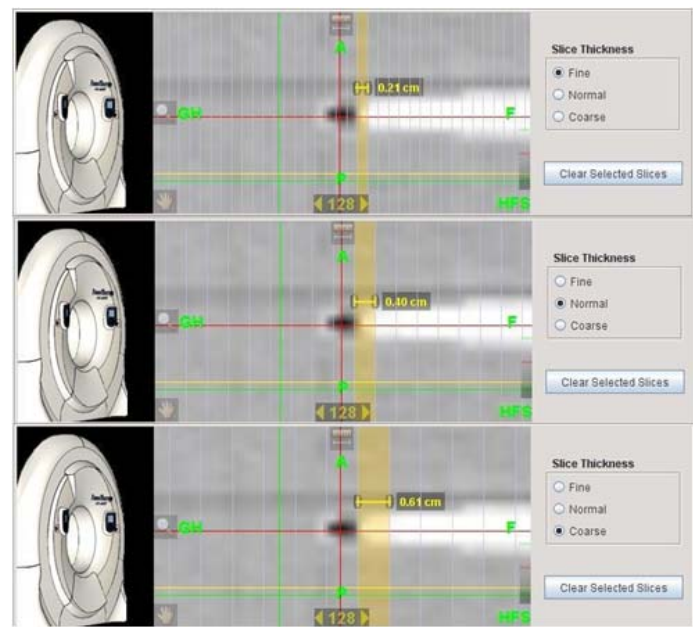

Fig.2. Slice thickness of Fine, Normal, Corse

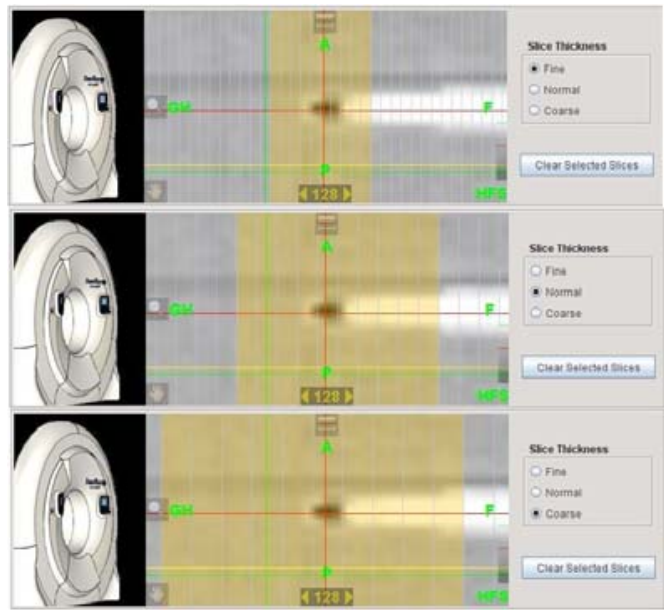

Fig.3. 10 Slice length by considering Fine, Normal, Corse slice

둘째, 동일한 Scan Volume (약 $9 \mathrm{~cm}$ )을 정한 뒤, 그에 따른 각 Slice Thickness (pitch 1,2,3 mm)별로 측정, 선 량차이를 비교하여, 총 3 번의 실험을 통해 평균값을 구하였다.

즉, pitch $1 \mathrm{~mm}$, scan slice 두께 $2.0 \mathrm{~mm}$ 로 체적의 길 
이 $9.0 \mathrm{~cm}$ 을 scan 할 경우, 총 scan slice는 45 개 이며, pitch $2 \mathrm{~mm}$, scan slice 두께 $4.0 \mathrm{~mm}$ 로 체적의 길이 9.0 $\mathrm{cm}$ 을 scan 할 경우, 총 scan slice는 22개 이고, pitch 3 $\mathrm{mm}$, scan slice 두께 $6.0 \mathrm{~mm}$ 로 체적의 길이 $9.0 \mathrm{~cm}$ 을 scan 할 경우, 총 scan slice는 15 개 이다.

각 Slice Thickness는 Fine-2mm, Normal-4mm, Coarse- $6 \mathrm{~mm}$ 의 값을 가지며, 각각의 Pitch는 Fine- $1 \mathrm{~mm}$, Normal-2 mm, Coarse-3 mm로 User가 바꿀 수 없는 제 조사의 고정된 값을 가진다. 이때 protocol 중 Fine과 Coarse는 각각 $2 \mathrm{~mm}, 6 \mathrm{~mm}$ 로 $9 \mathrm{~cm}$ 의 체적을 scan 할 때 45 개의 슬라이스와 15 개의 슬라이스를 만들 수 있 으나, Normal protocol인 경우, $4 \mathrm{~mm}$ 로 나누어떨어질 수 없기에 이때의 $\mathrm{scan}$ 체적은 $9 \mathrm{~cm}$ 이 아닌 $8.8 \mathrm{~cm}$ 로 선량 측정을 하였다. 이를 [그림 4.]에 나타내었다. 이 데이터를 바탕으로 각각 총 3 번의 측정을 통해 평균값 을 구하여, pitch $1 \mathrm{~mm}$ (Fine), pitch $2 \mathrm{~mm}$ (Normal), pitch $3 \mathrm{~mm}$ (Coarse) 간의 선량을 비교 분석해 보았다.

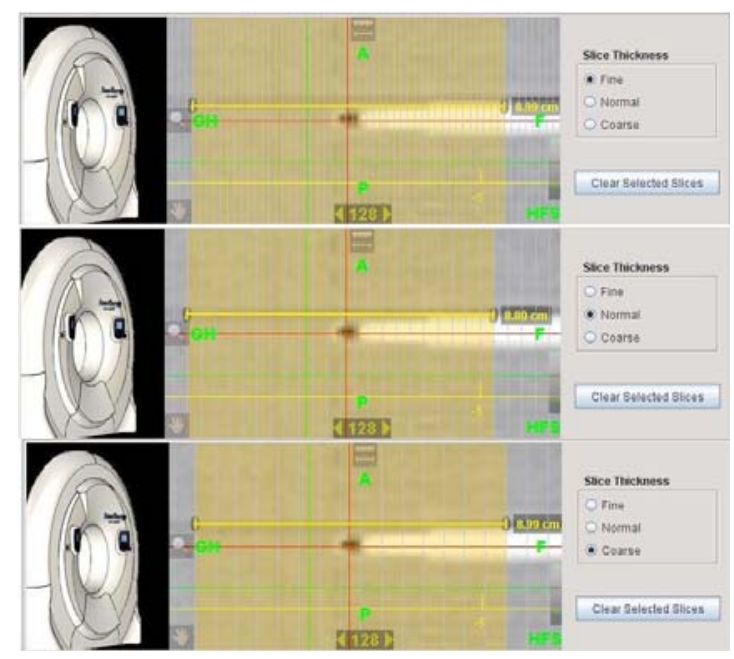

Fig.4. $9 \mathrm{~cm}$ length scan Slice number by considering Fine, Normal, Corse slice

\section{III. 결과}

측정결과, 각각의 pitch 마다 동일한 Slice 개수(10개) 로 Scan한 (이때의 scan length는 각각 2, 4, $6 \mathrm{~cm}$ ) 각각 의 평균값은, pitch $1 \mathrm{~mm}$ 일 때 $2.24 \mathrm{cGy}$, pitch $2 \mathrm{~mm}$ 일 때 $1.02 \mathrm{cGy}$, pitch $3 \mathrm{~mm}$ 일 때 $0.81 \mathrm{cGy}$ 가 측정되었 다. 만약 pitch $3 \mathrm{~mm}$ 를 기준으로 본다면, pitch $2 \mathrm{~mm}$ 는
$126 \%$ pitch $1 \mathrm{~mm}$ 는 $277 \%$ 선량이 더 들어갔고, pitch 2 $\mathrm{mm}$ 값을 기준으로 본다면 pitch $3 \mathrm{~mm}$ 는 $79.4 \%$ 로 약 $20.6 \%$ 선량이 덜 들어갔고, pitch $1 \mathrm{~mm}$ 는 $219 \%$ 선량이 더 들어간 것을 [표 1]에서 확인할 수 있다.

Table 1. Measurement of same slice scanning with respectively pitch value

\begin{tabular}{|c|c|c|c|c|c|c|c|c|}
\hline $\begin{array}{c}\text { condit io } \\
n\end{array}$ & pitch & length & 1st & 2nd & 3th & mean & $\%$ & $\%$ \\
\hline \multirow{3}{*}{$\begin{array}{l}\text { sane } \\
\text { slice }\end{array}$} & $1 \mathrm{~mm}$ & $(2.0 \mathrm{~cm}, 106)$ & 2.63 cas & 2.04 cay & 2.04 cay & 2.24 cay & 277 & 219 \\
\hline & $2 \mathrm{~mm}$ & $(4.0 \mathrm{~cm}, 108)$ & $1.02 \mathrm{cos} y$ & 1.02 cay & 1.02 o6y & 1.02 cay & 126 & 100 \\
\hline & $3 \mathrm{~mm}$ & $(6.0 \mathrm{~cm}, 108)$ & 0.80 cay & 0.82 cay & 0.81 cay & 0.81 cay & 100 & 79.4 \\
\hline
\end{tabular}

또한, 각 Slice Thickness 마다 일정한 Scan Volume(약 $9 \mathrm{~cm})$ 을 정하고 Scan한 각각의 평균값은 pitch $1 \mathrm{~mm}$ 일 때 $2.47 \mathrm{cGy}$, pitch $2 \mathrm{~mm}$ 일 때 $1.28 \mathrm{cGy}$, pitch $3 \mathrm{~mm}$ 일 때 $0.88 \mathrm{cGy}$ 가 측정되었다. 역시 pitch $3 \mathrm{~mm}$ 를 기준으 로 본다면, pitch $2 \mathrm{~mm}$ 는 $145.5 \%$, pitch $1 \mathrm{~mm}$ 는 $280 \%$ 선량이 더 들어갔고, pitch $2 \mathrm{~mm}$ 값을 기분으로 보면, pitch $3 \mathrm{~mm}$ 는 $68.8 \%$ 로 $31.2 \%$ 덜 들어갔고, pitch $1 \mathrm{~mm}$ 는 $193 \%$ 더 들어간 것을 [표 2]에서 확인 할 수 있다.

Table 2. Measurement of same volume scanning with respectively pitch value

\begin{tabular}{|c|c|c|c|c|c|c|c|c|}
\hline $\begin{array}{l}\text { condi } \\
\text { tion }\end{array}$ & pitch & length & ist & and & 3th & mean & $\%$ & $\%$ \\
\hline \multirow{3}{*}{$\begin{array}{c}\text { sane } \\
\text { volume }\end{array}$} & $1 \mathrm{~mm}$ & $(9.0 \mathrm{~cm}, 45 \mathrm{~s})$ & 2.54 cGy & 2.45 cay & 2.41 cay & $2.47 \mathrm{cGy}$ & 280 & 193 \\
\hline & $2 \mathrm{~mm}$ & $(8.8 \mathrm{~cm}, 22 \mathrm{~s})$ & $1.34 \mathrm{CG}$ & $1.26 \mathrm{coy}$ & 1.23 cay & $1.28 \mathrm{cG}$ & 145.5 & 100 \\
\hline & $3 \mathrm{~mm}$ & $(9.0 \mathrm{~cm}, 15 \mathrm{~s})$ & $0.87 \mathrm{cGy}$ & 0.88 cay & 0.89 cay & $0.88 \mathrm{cGy}$ & 100 & 68.8 \\
\hline
\end{tabular}

이것은 manufacturer tolerance에 나온 $4 \mathrm{cGy},{ }^{[8]}$ 선행 연구의 $\mathrm{MSAD}$ 값 1-3 cGy와 비슷한 양상의 선량이며, ${ }^{[9]}$ 각 Slice Thickness (pitch)간의 선량 차이는 Slice 두께와 Slice 개수에 따른 Scatter차이라고 볼 수 있다 ${ }^{[10]}$.

\section{IV. 고찰 및 결론}

영상 유도 기법은 방사선치료에서 표적의 위치 정 확도를 증가시키기 위해서는 필수적인 요소이며, 빈번 하지 않은 영상획득으로도 계통오차(systematic error)는 줄일 수 있으나, 임의 오차(random error)는 매일매일 
"Journal of the Korean Society of Radiology, Volume 7, Number 2"

획득하는 영상으로서만 줄일 수 있다는 특징이 있다.

일례로, 이비인후과 계통의 환자(Head \& Neck patient)는 이틀에 한 번씩 영상획득을 하였을 경우. 자 세 정렬 오차(set-up error)는 분할 조사 당 $10 \%$ 이상에 서 $5 \mathrm{~mm}$ 이상의 오차를 보인다는 결과와 ${ }^{[11]}$ 전립샘 암 환자를 이틀에 한 번씩 영상획득을 하였을 경우. 분할 조사 당 $20 \%$ 이상에서 $5 \mathrm{~mm}$ 이상의 오차를 보인다는 결과가 보고되었다 ${ }^{[7]}$. 이는 영상 획득의 중요성을 나 타내는 결과라 할 수 있지만 그에 따른 피폭선량의 증 가 역시 간과 할 수 없는 문제이므로 이를 측정하기 위해 본 논문에서는 각 Slice Thickness (pitch $1 \mathrm{~mm}, 2$ $\mathrm{mm}, 3 \mathrm{~mm}$ )간의 Point dose 차이를 비교해 본 결과, 최 대 3 배에 가까운 차이를 보였다. 환자의 Daily dose를 $200 \mathrm{cGy}$ 로 가정하였을 경우, 하루에 많게는 $1 \%$ 이상의 선량이 더 들어가는 셈이다. 이것을 전체 치료기간으 로 확대해보면 25-35번 치료받는 환자의 경우 50-70 $\mathrm{cGy}$ 정도의 선량이 더 들어가게 되는데 이는 결코 작 은 수치라고 할 수 없을 것이며 만약 치료 전, 후 2회 영상획득을 한다고 하면 선량은 100-140 cGy로 증가할 것이다 ${ }^{[7],[12]}$

결과적으로 보면 환자에게 직접적인 치료목적외의 부수적인 추가 선량은 pitch value, scanning length와 밀 접한 관계가 있으며, 이는 작은 pitch value와 큰 scanning 체적일수록 추가 선량을 증가시킨다는 것이 다. 또한 고정된 pitch 값을 사용하므로 비대한 환자가 왜소한 환자에 비해 더 적은 선량을, 같은 이유로 동 일한 환자일 경우 흉부와 복부의 선량이 체적이 작은 머리와 목에 비해 더 적은 선량을 받을 것이다.

Tomotherapy는 환자 치료 전에 MVCT Image를 획득 하고 이를 3 차원적으로 재구성 할 수 있음으로, 일반 적인 치료방법보다 더 정교하고 세밀한 방사선치료가 가능하다는 장점이 있다. 그러나 이로 인한 추가적인 피폭선량은 본 논문에서 알 수 있듯이 결코 무시 할 수 없다. 각 Slice Thickness마다 Image Quality의 차이는 존재한다. 각 병원마다 사용하는 Protocol은 다를 것이 고 치료하는 부위마다 사용하는 Protocol은 다를 것이 다. 환자치료에 있어서 정확성을 잃지 않으면서 최소 한의 피폭선량을 유지하기 위해서 각 Protocol간의 적 절한 선택이 무엇보다도 중요시 되어야 할 것이다.

\section{참고문헌}

[1] E. G. A. Aird, "Second cancer risk, concomitant exposure, and IRMER 2000." Br. J.Radol. 77, pp. 983-085, 2004

[2] Mackie T R, Holmes T, Swerdloff S, et al. "Tomotherapy: a new concept for the delivery of dynamic conformal radiotherapy." Med Phys 20, pp. 1709-1719, 1993

[3] Lisa J. Forrest, Thomas Rockwell Mackie, Ken Ruchala, et al. "The utility of megavoltage computed tomography image from a helical tomotherapy system for set-up verification purposes." Int J Radiat Oncol Biol Phys 60, pp. 1639-1644, 2004

[4] Rajesh A. Kinhikar, Zubin Master, Dipak S. Dhote, and Deepak D. Deshpande, "Initial dosimetric experiience with megavoltage computed tomography detectors and estimation of pre and postrepair dosimetric parameters of a first helical Hi-Art II tomotherapy machine in india." Med Phys 34, pp.73-79, 2009

[5] Ruchala KJ, Olivera GH, Schloesser et al. "Megavoltage CT on a tomotherapy system." Phys Med Biol 44:pp. 2597-2621, 1999

[6] Liu B, Bai M, Fei XL, et al. "Optimization of patient dose parameters of CT examination." Zhong Guo Yi Liao Zhuang Bei 23(3), pp. 54-56, 2008

[7] Amish P. Shah, Katja M. Langen, Kenneth J. Ruchala, et al. "Patient dose from megavoltage computed tomography imaging." Int J. Radiation Oncology Biol. Phys, Vol. 70, No. 5, pp. 1579-1587, 2008

[8] Acceptance Test Procedures and Dosimetry Guide. Tomotherapy Inc: 2005

[9] Mackie TR, Balog J, Ruchala K, et al. "Tomotherapy Semin" Radiat Oncol 9, pp. 108-117, 1999

[10] Sanford L. Meeks, Joseph F. Harmon, Katja M. Langen, et al. "Performance characterization of megavoltage computed tomography imaging on helical tomotherapy unit." Med Phys 32, pp. 2673-2681, 2005

[11] Zeidan O A, Langen K M, Meeks S L, et al. "Evaluation of image guidance protocols in the treatment of head and neck cancers." Int J Radiat Oncol Biol Phys 67, pp. 670-677, 2007

[12] Beavis A W, "Is tomotherapy the future of IMRT?" $\mathrm{Br} \mathrm{J}$ Radio, 77(916), pp. 285-295, 2004 